\title{
Los médicos de atención primaria no tienen en cuenta el riesgo cardiovascular para decidir la intensidad del tratamiento de la presión arterial
}

Primary care physicians do not consider cardiovascular risk to decide of antihypertensive therapy's intensity

\section{Objetivo}

Investigar si los médicos de atención primaria consideran el riesgo cardiovascular $(\mathrm{RCV})$ individual además del nivel de presión arterial (PA) al seleccionar una estrategia terapéutica; y evaluar su efectividad en el manejo clínico de la hipertensión arterial (HTA) para determinar los predictores de control e intensidad del tratamiento.

\section{Diseño}

Estudio de corte transversal, retrospectivo.

\section{Lugar}

Unidad de Medicina Familiar y Preventiva, Hospital Italiano de Buenos Aires, Argentina.

\section{Pacientes}

922 pacientes hipertensos seleccionados en forma aleatoria de 14000 historias clínicas.

\section{Evaluación de factores pronósticos}

Tasas de control de HTA, reducción (en $\mathrm{mmHg}$ ) de la presión sistólica y diastólica e intensidad del tratamiento, estratificadas por estadíos y grupos de RCV.

\section{Resultados principales}

Según los criterios del Sexto Consenso de Tratamiento de la Hipertensión de EE.UU (JNC VI) los pacientes se distribuyeron en: estadío I $38,4 \%$, II $38,5 \%$ y III $22,5 \%$. Solo $12,5 \%$ de los pacientes no tenía otros factores de riesgo, daño de órgano blanco o enfermedad cardiovascular (grupo A); $48,6 \%$ tenía por lo menos otro factor de riesgo (grupo B) y $39,3 \%$ tenía daño de órgano blanco, diabetes o enfermedad cardiovascular (grupo C). El 53,3\% estaba bien controlado (menos de 140/90 mmHg). El mejor control se alcanzó entre los pacientes con Estadío I $(62,2 \%)$ y disminuyó en los II y III respectivamente $(53,1$ y $39,1 \%)$. No hubo diferencias en el grado de control de la PA entre los diferentes grupos de riesgo (A: $58,6 \%$, B: $51,3 \%$ y C $54,4 \%$ ) ni en el grado de descenso de la PA sistólica o diastólica. Se evaluó la intensidad del tratamiento en 590 pacientes (64\%). En los estadíos mas avanzados fue mayor y no hubo diferencias según los grupos de riesgo. El estadío inicial fue el único predictor de control y no lo fueron la estratificación del grupo de riesgo, el tiempo de seguimiento, el número de visitas al médico, la intensidad del tratamiento, el sexo, ni la edad. Para la intensidad del tratamiento, el estadío inicial y el tiempo de seguimiento fueron los únicos predictores. A mayor estadío inicial y mayor el tiempo de seguimiento, mayor fue la intensidad del tratamiento.

\section{Conclusiones}

El grado de control de la PA está en estrecha relación con el nivel de la PA y los médicos no parecen considerar el RCV en las decisiones terapéuticas.

Palabras clave: hipertensión arterial, terapéutica, calidad de atención Key words: therapeutics, hypertension, quality of care.

Fuente de financiamiento: Unidad de Medicina Familiar y Preventiva, Hospital Italiano de Buenos Aires, Argentina.

\section{Comentario}

La HTA afecta aproximadamente a diez millones de argentinos adultos y es un factor de riesgo mayor para infarto agudo de miocardio, accidente cerebrovascular, insuficiencia cardiaca e insuficiencia renal. EI RCV no solo está determinado por el nivel de PA, sino también por la presencia de daño de órgano blanco y otros factores de riesgo. Numerosas guías para el manejo de la HTA enfatizan la importancia de la estratificación del RCV en la toma de decisiones clínicas.

El tratamiento de la HTA ha demostrado claramente que una pequeña reducción en la PA reduce la morbilidad y mortalidad cardiovascular y renal.

El grado de control de la PA en el estudio analizado es elevado, si se lo compara con los datos de prevalencia $(29,3 \%)$ conocimiento $(66,5 \%)$ tratamiento $(53,7 \%)$ y control $(33,1 \%)$ de la HTA en adultos norteamericanos entre 1999 y $2004^{1}$.
Por otra parte, y teniendo en cuenta que se trata de un estudio retrospectivo basado en la revisión de historias clínicas, podría haber un sesgo* en relación a una insuficiente o incorrecta documentación de los datos, o en la selección de los pacientes ya que pertenecían a un sistema de medicina prepaga con cuidados de atención primaria de la salud lo que podría condicionar una mayor adherencia a la terapéutica.

\section{Conclusiones del comentador}

Se refuerza la necesidad de incorporar la evaluación y medición del RCV en el paciente hipertenso individual para mejorar la calidad del cuidado de la salud cardiovascular.

Gabriel Waisman [ Jefe de Servicio de Clínica Médica y de la Sección Hipertensión Arterial del Hospital Italiano de Buenos Aires. ]

Waisman G. Los médicos de atención primaria tratan a los hipertensos según el nivel de presión arterial, sin tener en cuenta su riesgo cardiovascular. Evid. actual. práct. ambul; 10(2): 44, mar-abr.2007. Comentado de: Gimpel N, Schoj V, Rubinstein A. Quality management of hypertension in primary care: Do physicians treat patients' blood pressure level or cardiovascular risk? Quality in Primary Care 2006; 14:211-17.

\section{Referencias}

1. Kwok Leung Ong, Bernard M.Y. Cheung, Yu Bun Man, Chu Pak Lau, Karen S.L. Lam Prevalence, Awareness, Treatment, and Control of Hypertension Among United States Adults 1999-2004. Hypertension. 2007; 49:69-75. 